\title{
The anomalous 0.5 and 0.7 conductance plateaus in quantum point contacts
}

\author{
Henrik Bruus, Vadim V. Cheianov, and Karsten Flensberg \\ Ørsted Laboratory, Niels Bohr Institute, Universitetsparken 5, DK-2100 Copenhagen Ø, Denmark
}

(Physica E 10, 97, (2001))

\begin{abstract}
The anomalous 0.5 and 0.7 conductance plateaus in quantum point contacts in zero magnetic field are analyzed within a phenomenological model. The model utilizes the Landauer-Büttiker formalism and involves enhanced spin correlations and thermal depopulation of spin subbands. In particular we can account for the plateau values 0.5 and 0.7 , as well as the unusual temperature and magnetic field dependences of the 0.7 plateau. Finally, the model predicts the possibility of coexisting 0.5 and 0.7 plateaus.
\end{abstract}

\section{Keywords: quantum point contacts, anomalous conductance, 0.5 plateau, 0.7 plateau}

\section{INTRODUCTION}

It has been known and well understood since 1988 [1-3] that the dc-conductance $G$ of narrow quantum point contacts and quantum wires (both referred to as QPCs below) is quantized in units of $G_{2}=2 e^{2} / h$. During the past five years an increasing part of the experimental and theoretical work on QPCs has been devoted to studies of a particular deviation from this integer quantization known as 0.7 conductance anomaly 4414 . This anomaly is a narrow plateau, or in some cases just a plateau-like feature appearing in scans of $G$ versus gate voltage $V_{g}$ at a value of $G$ which is reduced by a factor 0.7 relative to the ideal value $G_{2}$. The 0.7 anomaly has been recorded in numerous QPC transport experiments (even before it was noted in 1996, see e.g. Ref. 11). Recently the appearance of an anomalous plateau at the value $0.5 G_{2}$ was also reported 10,11.

In this paper we show that many of the experimental findings regarding the 0.5 and 0.7 anomalous plateaus can be consistently interpreted by invoking the model of enhanced spin correlations in the QPC, which we previously put forward to explain the 0.7 plateau 114. We emphasize that our model does not rely on the existence of static polarization, which would be inconsistent with some general theorems [19], but rather on effects of dynamical local polarization in the QPC.

Already in the first paper [4] it was pointed out that due to its magnetic field dependence the 0.7 anomaly may be related to spontaneous spin polarization of electrons in the QPC. Theoretical attempts to link the 0.7 anomaly to spontaneous spin polarization have been made [12,13]. However, none of these approaches have explained all of the experimental facts, and most strikingly they failed to predict the observed plateau at $0.7 G_{2}$. Also the 0.7 anomaly cannot be explained by impurity backscattering mechanisms, Luttinger liquid effects or an interplay of both, the predicted temperature dependence being opposite to the observed one.

\section{SUMMARY OF EXPERIMENTAL FACTS}

In summarizing experimental data we will mainly refer to the work of the Cambridge group [4] 6] and the Copenhagen group [7 9] presenting detailed studies of the magnetic field and temperature dependence of the 0.7 anomaly.

The main experimental features of the 0.7 anomaly are:

(e1) The anomalous plateau is observed in a large variety of QPCs at a value $G=\gamma G_{2}$, where the suppression factor $\gamma$ is close to 0.7 .

(e2) The temperature dependence is qualitatively the same for all samples: the anomalous plateau is fully developed in some (device dependent) temperature range typically above $2 \mathrm{~K}$. With increasing temperature both the anomalous and the integer plateaus vanish by thermal smearing, while with decreasing temperature the width of the anomalous plateau shrinks the conductance approaching $2 G_{2}$. 专 9].

(e3) A detailed study of the temperature dependence of $\gamma$ shows that in the low temperature regime the conductance suppression has an activated behavior: $1-\gamma(T) \propto$ $\exp \left(-T_{a} / T\right)$ [8,9].

(e4) The activation temperature $T_{a}$ is a function of $V_{g}$ vanishing at some critical gate voltage $V_{g}^{0}$. Close to $V_{g}^{0}$ the dependence of $T_{a}$ on $V_{g}$ is well approximated by a power law [8.9], $T_{a} \propto\left(V_{g}-V_{g}^{0}\right)^{\alpha}$, with $\alpha \approx 2$.

(e5) At a fixed temperature corresponding to a well developed 0.7 plateau, $\gamma$ shows a strong dependence on an in-plane magnetic field [4 6]. With increasing magnetic field $\gamma$ smoothly decreases from 0.7 at $B=0 \mathrm{~T}$ to 0.5 at $B=13 \mathrm{~T}$.

(e6) Under the same temperature conditions as in (e5) the 0.7 anomaly depends on the source-drain bias. The suppression factor $\gamma$ increases smoothly from $\sim 0.7$ at zero bias to $\sim 0.9$ at large bias $(\sim 2 \mathrm{mV})[9]$.

(e7) In some samples the anomalous plateau was reported to appear at $G=0.5 G_{2}$ [10,11] rather than at $G=0.7 G_{2}$. 
(a)

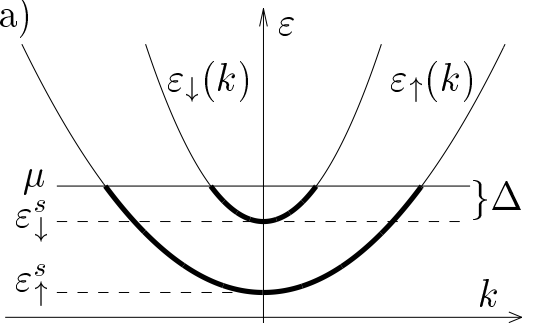

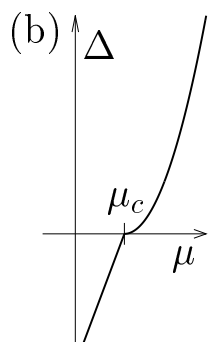

FIG. 1. (a) The instantaneous spin split subband structure of our model. (b) The functional form Eqs. (值) and (5) of $\Delta(\mu)=\mu-\varepsilon_{\downarrow}^{s}(\mu)$ giving rise to the anomalous 0.7 plateau.

\section{THE PHENOMENOLOGICAL MODEL}

The ground state of the two-dimensinal electron gas (2DEG) has been studied extensively using ever more refined methods involving local density functional theory [15, 16] and Monte Carlo calculations [17]. In particular it is argued in the latter work that at at zero temperature there exist at least three phases of the 2DEG: an unpolarized fluid $\left(r_{s}<13\right)$, a fully spin polarized fluid $\left(13<r_{s}<33\right)$, and a Wigner crystal polarization $\left(r_{s}>33\right)$. More recently, also using density functional theory, the quasi-1d case has been studied and evidence for a spin polarization has been found [12, 18]. In Ref. [12] the spin polarization in QPC's was studied at zero temperature assuming that the expression in the local-density approximation for the exchange energy of the bulk 2DEG is also valid in narrow quasi-1d constrictions. This work suggested the possibility of spin polarization in QPC's, but yielding only plateaus at 0.5 and 1.0, it failed to explain the 0.7 anomaly. We base our phenomenological model on these previous works on spin polarization. Without refering to any particular model for the exchange energy, we simply assume the existence on spin polarization expressed by a general spin-density functional involving three unknown constants that are to be determined experimentally.

We stress that the spin polarization not necessarily is permanent. It may well exhibit mesoscopic fluctuations. It is in fact enough to assume that the dynamics of the spin degrees of freedom in the constriction is adiabatic (slow) as compared to both the length of the QPC and the time of passage of electrons through the QPC. Then, given some instantaneous spin configuration the transmission coefficient $\mathcal{T}_{\sigma}^{\text {tot }}$ for a spin- $\sigma$ electron going through the QPC can thus be calculated as $\mathcal{T}_{\sigma}^{\text {tot }}=\mathcal{T}_{\sigma}(E) P_{\sigma}+\mathcal{T}_{\bar{\sigma}}(E) P_{\bar{\sigma}}$. Here $P_{\sigma}\left(P_{\bar{\sigma}}\right)$ is the probability of finding the incoming spin parallel (antiparallel) to the instantaneous polarization. In the isotropic case with $P_{\sigma}=P_{\bar{\sigma}}$ this leads to the same results as a static situation where two spin subbands are formed. For simplicity we just treat the case of a stationary polarization in the following and derive the subband structure shown in Fig. 1a.
This discussion of spin-polarization serves as the justification of the basic assumption in our work: the existence of a "critical" chemical potential $\mu_{c}$, where the cross-over from full to partial polarization occurs. For $\mu<\mu_{c}$ the densities are $n_{\uparrow}=n_{\uparrow}^{0}$ and $n_{\downarrow}=0$, while for $\mu>\mu_{c}$ a non-zero $n_{\downarrow}$ develops, i.e. two spin subbands appears with different subband edges $\varepsilon_{\sigma}^{s}$ as depicted in Fig.1a. It turns out that all the phenomenology of the anomalous plateaus is contained in the $\mu$ dependence of the position of the minority subband edge $\varepsilon_{\downarrow}^{s}(\mu)$ relative to $\mu$. This important parameter is denoted $\Delta(\mu)$ :

$$
\Delta(\mu)=\mu-\varepsilon_{\downarrow}^{s}(\mu) .
$$

Our entire analysis is based on the local spin density functional written as

$$
F=E\left[n_{\downarrow}, n_{\uparrow}\right]-\mu\left(n_{\downarrow}+n_{\uparrow}\right) .
$$

Near the critical point $\mu_{c}$, for $\mu>\mu_{c}$, we have $n_{\downarrow} \ll n_{\uparrow}$ and the condition for the minimum of the free energy becomes

$$
\begin{aligned}
& \frac{\partial F}{\partial n_{\uparrow}}=\alpha+\alpha^{\prime} \delta n_{\uparrow}+\gamma n_{\downarrow}-\mu=0, \\
& \frac{\partial F}{\partial n_{\downarrow}}=\beta+\beta^{\prime} n_{\downarrow}+\gamma \delta n_{\uparrow}-\mu=0 .
\end{aligned}
$$

We have made the linearization $n_{\uparrow}=n_{\uparrow}^{0}+\delta n_{\uparrow}$ for the majority spins and assumed that the energy functional $F$ near the minimum is bilinear in $\delta n_{\uparrow}$ and $n_{\downarrow}$.

The solution for the minority spin density in the case of $\mu>\mu_{c}$ is $n_{\downarrow} \propto\left(\mu-\mu_{c}\right)$ which combined with the $1 \mathrm{~d}$ property that $n_{\downarrow}^{2} \propto \varepsilon_{F}^{\downarrow}$. But $\varepsilon_{F}^{\downarrow}=\mu-\varepsilon_{\downarrow}^{s}=\Delta$, and we arrive at:

$$
\Delta(\mu)=C\left(\mu-\mu_{c}\right)^{2}, \quad \text { for } \mu>\mu_{c} .
$$

For $\mu<\mu_{c}$ we have $n_{\downarrow}=0$ and $\Delta(\mu)$ is now the excitation gap for flipping a spin at the Fermi level, i.e. $\Delta(\mu)=$ $\left(\partial_{n_{\uparrow}} F-\partial_{n_{\downarrow}} F\right)=0$, which gives $\Delta(\mu)=\beta+\gamma \delta n_{\uparrow}$. This combined with $\delta n_{\uparrow} \propto \mu-\mu_{c}$ leads to:

$$
\Delta(\mu)=D\left(\mu-\mu_{c}\right), \quad \text { for } \mu<\mu_{c} .
$$

We have thus derived the dispersion laws depicted in Fig. 1:

$$
\begin{aligned}
& \varepsilon_{\uparrow}(k)=\frac{\hbar^{2}}{2 m} k^{2}+\varepsilon_{\uparrow}^{s}, \\
& \varepsilon_{\downarrow}(k)=\frac{\hbar^{2}}{2 m} k^{2}+\mu-\Delta(\mu) .
\end{aligned}
$$

Given these dispersion relations, at finite temperature $T$ using an idealized step-function transmission coefficient the LB conductance $G(T)$ of this system is [3]

$$
G(T)=\frac{1}{2} G_{2} \sum_{\sigma=\uparrow, \downarrow} \int_{-\infty}^{\infty} d \varepsilon \Theta\left(\varepsilon-\varepsilon_{\sigma}^{s}\right)\left(-f^{\prime}[\varepsilon-\mu]\right),
$$

where $f^{\prime}$ is the derivative of the Fermi-Dirac distribution $f[x]=\left[\exp \left(x / k_{B} T\right)+1\right]^{-1}$ and $\Theta(x)$ is the step function. By integration and using Eq. (11) we obtain 

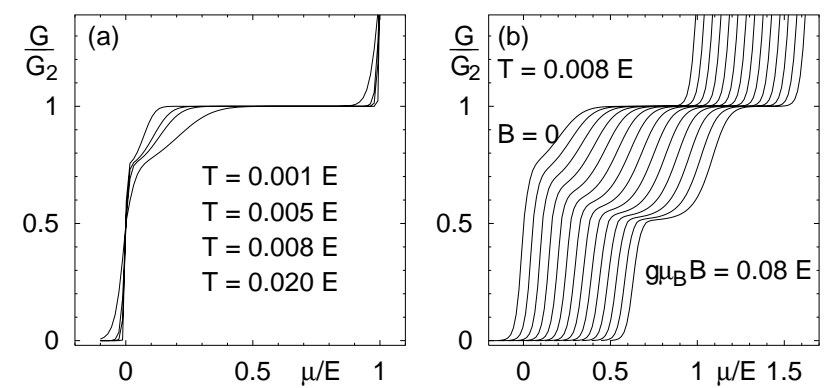

FIG. 2. (a) The conductance from Eqs. (8) and (4) with $C=0.5, D=1.0$ and $\mu_{c}=\varepsilon_{\uparrow}^{s}$. All energies are given in units of the transverse mode subband spacing $E$. (b) The conductance as in (a) but with fixed $T=0.008 E$ and an applied in-plane magnetic field varying from 0 to $0.08 \mathrm{E} / \mathrm{g} \mu_{B}$. For clarity the curves in (b) have been offset horizontally.

$$
G(T)=\frac{1}{2} G_{2}\left(f\left[\varepsilon_{\uparrow}^{s}-\mu\right]+f[-\Delta(\mu)]\right) .
$$

All predictions relating to experiments on the conductance of QPC's follow from this simple analytical form.

Before turning to a thorough analysis of this expression we note some of its basic features. Consider first the situation where the spin polarization is nearly complete, i.e. $\Delta(\mu) \ll \varepsilon_{\downarrow}^{s}(\mu)-\varepsilon_{\uparrow}^{s}(\mu)$. In this case, at low temperatures, $k_{B} T \ll \Delta(\mu)$, both terms in Eq. (8) are 1 and the conductance is the usual $G_{2}$. However, in the temperature range

$$
\Delta(\mu) \ll k_{B} T \ll \varepsilon_{\downarrow}^{s}(\mu)-\varepsilon_{\uparrow}^{s}(\mu),
$$

the contribution of the first term is 0.5 while the second term remains 1 yielding $G=0.75 G_{2}$. Due to the parabolic $\mu$-dependence of $\Delta$ given by Eq. (何), which in a sense pins $\varepsilon_{\downarrow}^{s}$ to $\mu$, the condition (9) is in fact fulfilled for a sufficiently broad range of $\mu$ (in experiments $\mu \propto V_{g}$ ), thus giving rise to a 0.7 quasi-plateau.

An in-plane magnetic field $\mathbf{B}$ is readily taken into account by adding Zeeman energy terms and substituting

$$
\varepsilon_{\uparrow}^{s} \rightarrow \varepsilon_{\uparrow}^{s}-g \mu_{B}|\mathbf{B}|, \quad \varepsilon_{\downarrow}^{s} \rightarrow \varepsilon_{\downarrow}^{s}+g \mu_{B}|\mathbf{B}| .
$$

\section{EXPERIMENTAL IMPLICATIONS OF THE MODEL}

In the following we discuss how the model can explain the experimental observations (e1)-(e6). In Fig. 2(a) observations (e1) and (e2) are clearly seen in the model calculation. In this idealized case with a step-function transmission coefficient the plateau appears at 0.75 as discussed above. Observation (e3) follows trivially from Eq. (7) with the activation temperature $T_{a}=\Delta(\mu)$. Assuming that in the vicinity of $\mu_{c}$ the chemical potential depends linearly on the gate voltage $V_{g}$ Eq. (4) immediately predicts (e4) with the exponent $\alpha=2$.
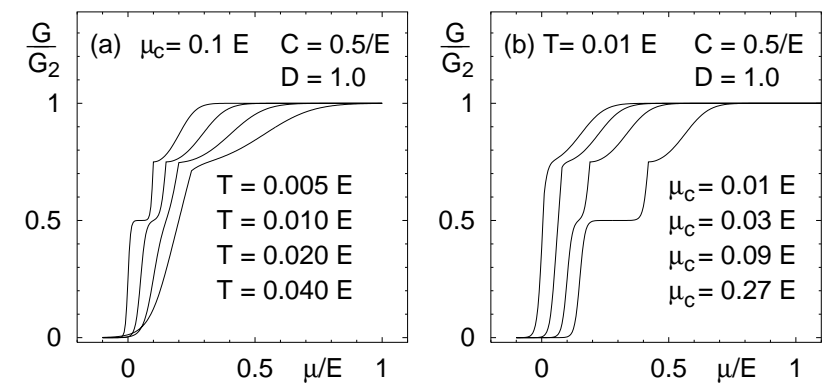

FIG. 3. (a) $G$ for fixed $\mu_{c}$ and coefficient $C$ while changing temperature. For clarity, the curves are offset, the temperature increasing from left to right. Note the disappearence of the 0.5 plateau at high $T$. (b) $G$ for fixed temperature and coefficient $C$ while changing $\mu_{c}$. For clarity, the curves are offset, $\mu_{c}$ increasing from left to right. Note the disappearence of the 0.5 plateau for small values of $\mu_{c}$.

The result of the model calculation in the presence of a magnetic field using Eqs. (8), (41) and (10) is shown in Fig. 2(b). In accordance with observation (e5) the 0.7 anomaly develops smoothly into an ordinary Zeeman split 0.5 plateau. The experimental observation (e6) concerns finite bias. This brings us into a strong nonequilibrium situation which is outside the scope of the present work. However, considering a small finite bias not too far from the equilibrium case, we do find that the 0.75 plateau rises, which gives additional support for the picture presented here. In Ref. [9] the finite bias measurements were well explained by straightforward extension of our model to the non-linear regime.

Apart from 0.7 quasiplateau, our model also predicts a plateau at $0.5 G_{2}$, which should be seen in perfect QW/PCs at zero temperature and zero magnetic field when $\varepsilon_{\uparrow}^{s}<\mu<\mu_{c}$ (full polarization). In order to observe the 0.5 plateau the condition $k_{B} T<\min \left\{|\Delta|, \mu-\varepsilon_{\uparrow}^{s}\right\}$ must be fulfilled. Otherwise thermal smearing will destroy the plateau. Note that even if the 0.5 plateau is thermally smeared, Eq. (9) may still hold and result in a 0.7 plateau. This is illustrated in Fig. 3. In Fig. 3(a) $\mu_{c}$ is fixed and the temperature is changed, while in Fig. 3(b) the value of $\mu_{c}$ is changed at a fixed temperature. One can see that in a situation where $T<\mu_{c} / 8$ the 0.5 and the 0.7 plateaus can be seen simultaneously. This situation has never been observed experimentally, which might be an indication that the experimentally accessible temperatures are too high to resolve the 0.5 plateau. However according to (e7), a situation where 0.5 plateau appeared without a 0.7 plateau was observed. This might happen if the range of $\mu$ corresponding to partial polarization in the QPC was particularly narrow as compared to that corresponding to full polarization. In terms of the parameters of our model it would mean e.g. a very large constant $C$.

The role of parameter $C$ is illustrated in Fig. 4. One can see that with increasing $C$ the 0.7 quasiplateau be- 

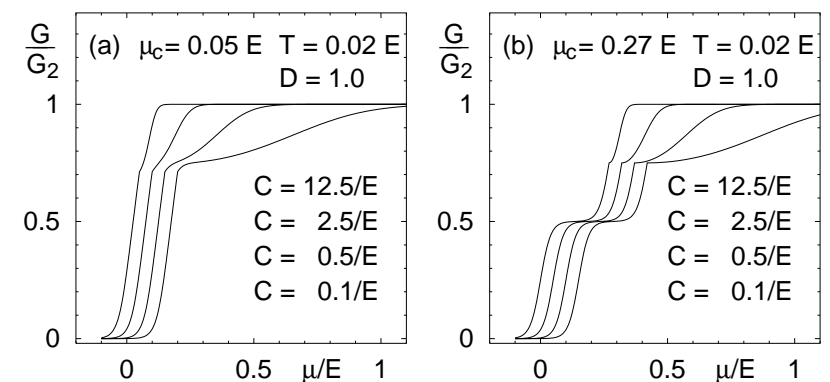

FIG. 4. (a) $G$ for fixed temperature and a small value of $\mu_{c}$ while changing the coefficient $C$. For clarity, the curves are offset, $C$ decreasing from left to right. Note the emerging 0.7 plateau at small $C$. (b) The same as in (a) except for a larger value of $\mu_{c}$. Note the coexistence of the 0.5 and 0.7 plateaus.

comes narrower and less prominent, and practically disappears in comparison with the 0.5 at very large values of $C$.

\section{NON-IDEAL TRANSMISSION}

In our idealized model we used a step-function transmission coefficient for electrons traversing the QPC. Nonideal transmission is easily taken into account by replacing the theta function in Eq. (7) with a given transmission coefficient $\mathcal{T}_{\sigma}(\varepsilon)$ :

$$
G(T)=\frac{1}{2} G_{2} \sum_{\sigma=\uparrow, \downarrow} \int_{-\infty}^{\infty} d \varepsilon \mathcal{T}_{\sigma}(\varepsilon)\left(-f^{\prime}[\varepsilon-\mu]\right) .
$$

Through the transmission coefficient $\mathcal{T}_{\sigma}(\varepsilon)$ the conductance now depends on the geometry of the QPC and is no more universal. However, some qualitative predictions can still be made without a knowledge of the exact transmission properties of the QPC. First of all, due to the conditions Eqs. (9) and (19) the quasi-plateau persists. Secondly, mainly the transmission coefficient of minority spin band is affected which results in a suppression of the anomalous plateau while the integer plateau remains close to 1 . Model calculations show that the anomalous 0.7 plateau may be suppressed to the values of $G=0.6 G_{2}$ without being destroyed [14]. Another effect of non-ideal transmission is the "smearing" of the $G$ vs. $\mu$ dependence as compared to the situation of ideal transmission. The character of smearing is not unlike the thermal smearing, and it may e.g. contribute to the effect of masking the 0.5 plateau in the samples where only the 0.7 plateau is seen.

\section{SUMMARY AND CONCLUSION}

We have presented a phenomenological model which can account for the experimental observations of the anomalous 0.7 and 0.5 conductance plateaus in mesoscopic QPCs. The model is built on an assumption of an effective instantaneous partial polarization seen by the traversing electrons, while the ground state itself needs not have a finite magnetic moment. Even in its simplest form excluding effects of non-ideal transmission our model is capable of providing a very satisfactory description of different experimental situations, which is achieved by varying the two model parameters $\mu_{c}$ and $C$.

\section{Acknowledgements}

The authors acknowledge support from the Danish Natural Science Research Council through Ole Rømer Grant No. 9600548.

[1] B.J. van Wees et al., Phys.Rev.Lett. B 60, 848 (1988).

[2] D.A. Wharam et al., J.Phys.C 21, L209 (1988).

[3] for a review see e.g. H. Van Houten, C.W.J. Beenakker, and B. van Wees, p. 9 in Nanostructured Systems, M. Reed vol. ed., Semiconductors and Semimetals 35, R.K. Williamson, A.C. Beer and R. Weber eds., Academic Press, 1992.

[4] K.J. Thomas et al., Phys. Rev. Lett. 77, 135 (1996).

[5] K.J. Thomas et al., Phys. Rev. B 58, 4846 (1998).

[6] C.-T. Liang et al., Phys. Rev. B 60, 10687 (1999).

[7] A. Kristensen et al., J. Appl. Phys. 83, 607 (1998).

[8] A. Kristensen et al., Physica B 249-251, 180 (1998).

[9] A. Kristensen et al., Phys. Rev. B 62, 10950 (2000).

[10] K.J. Thomas et al., Phys. Rev. B 61, R13365 (2000);

[11] D. J. Reilly et al., Phys. Rev. B 63, R121311 (2001).

[12] C.-K. Wang and K.-F. Berggren, Phys. Rev. B 57, 4552 (1998).

[13] B. Spivak and F. Zhou, Phys. Rev. B 61, 16730 (2000).

[14] H. Bruus, V. Cheianov, K. Flensberg, cond-mat/0002338.

[15] F. Stern, Phys. Lett. 30, 278 (1973).

[16] F. Stern and S. D. Sarma, Phys. Rev. B 30, 840 (1984).

[17] D. Ceperley, Phys. Rev. B 18, 3126 (1978).

[18] C.-K. Wang and K.-F. Berggren, Phys. Rev. B 54, R14257 (1996).

[19] E. Lieb and D. Mattis, Phys. Rev. 125, 164 (1962); N.D. Mermin and H. Wagner, Phys. Rev. Lett. 17, 1133 (1966). 